\title{
Matlis flat modules
}

\author{
C. Selvaraj ${ }^{1}$ and P. Prabakaran ${ }^{2}$
}

Department of Mathematics, Periyar University, Salem - 636 011, TN, India

E-mail: selvavlr@yahoo. $\mathrm{com}^{1}$, prabakaranpvkr@gmail. $\mathrm{com}^{2}$

\begin{abstract}
In this paper, we introduce Matlis flat modules as a generalization of copure flat modules and give their characterizations. We prove that if $R$ is a commutative Artinian ring and $S \subset R$ is a multiplicative set, then $S^{-1} M$ is a Matlis flat $S^{-1} R$-module for any Matlis flat $R$-module $M$. Also we prove that every module has Matlis flat preenvelope over commutative Artinian rings.
\end{abstract}

2010 Mathematics Subject Classification. 16D10. 16D50, 16E30

Keywords. Injective envelope, Matlis flat module, Matlis injective module, Matlis flat preenvelope.

\section{Introduction}

Throughout this paper, $R$ denotes an associative ring with identity and all modules are unitary. Denote by $R$-Mod the category of left $R$-modules and by Mod- $R$ the category of right $R$-modules. As usual, $p d_{R}(M), i d_{R}(M)$, and $f d_{R}(M)$ will denote the projective, injective and flat dimensions of a left $R$-module $M$, respectively. The character module $\operatorname{Hom}_{\mathbb{Z}}(M, \mathbb{Q} / \mathbb{Z})$ is denoted by $M^{+}$and for a class of $R$-modules $\mathcal{C}$, we denote by $\mathcal{C}^{+}=\left\{C^{+} \mid C \in \mathcal{C}\right\}$. For unexplained concepts and notations, we refer the reader to [4], [15] and [19].

We first recall some known notions and facts needed in the sequel.

Let $\mathcal{C}$ be a class of left $R$-modules and $M$ a left $R$-module. Following [4], we say that a map $f \in \operatorname{Hom}_{R}(C, M)$ with $C \in \mathcal{C}$ is a $\mathcal{C}$-precover of $M$, if the group homomorphism $\operatorname{Hom}_{R}\left(C^{\prime}, f\right)$ : $H_{o m}\left(C^{\prime}, C\right) \rightarrow \operatorname{Hom}_{R}\left(C^{\prime}, M\right)$ is surjective for each $C^{\prime} \in \mathcal{C}$. A $\mathcal{C}$-precover $f \in H_{o m}(C, M)$ of $M$ is called a $\mathcal{C}$-cover of $M$ if $f$ is right minimal, that is, if $f g=f$ implies that $g$ is an automorphism for each $g \in \operatorname{End}_{R}(C)$. $\mathcal{C} \subseteq R$-Mod is a precovering class (covering class) provided that each module has a $\mathcal{C}$-precover $(\mathcal{C}$-cover $)$. Dually, we have the definition of $\mathcal{C}$-preenvelope $(\mathcal{C}$-envelope $)$. In general, $\mathcal{C}$-covers $(\mathcal{C}$-envelopes) may not exist, if exists, they are unique up to isomorphism.

Given a class $\mathcal{C}$ of left $R$-modules, we write

$$
\begin{aligned}
& \mathcal{C}^{\perp}=\left\{M \in R \text {-Mod } \mid \operatorname{Ext}_{R}^{1}(C, M)=0, \quad \forall C \in \mathcal{C}\right\} \\
& { }^{\perp} \mathcal{C}=\left\{M \in R \text {-Mod } \mid \operatorname{Ext}_{R}^{1}(M, C)=0, \quad \forall C \in \mathcal{C}\right\} .
\end{aligned}
$$

A pair $(\mathcal{F}, \mathcal{C})$ of classes of right $R$-modules is called a cotorsion theory [4] if $\mathcal{F}^{\perp}=\mathcal{C}$ and ${ }^{\perp} \mathcal{C}=\mathcal{F}$. A cotorsion theory $(\mathcal{F}, \mathcal{C})$ is called perfect $[5]$ if every right $R$-module has a $\mathcal{C}$-envelope and an $\mathcal{F}$ cover. A cotorsion theory $(\mathcal{F}, \mathcal{C})$ is called hereditary [5] if whenever $0 \rightarrow L^{\prime} \rightarrow L \rightarrow L^{\prime \prime} \rightarrow 0$ is exact with $L, L^{\prime \prime} \in \mathcal{F}$, then $L^{\prime}$ is also in $\mathcal{F}$. By [5, Proposition 1.2], $(\mathcal{F}, \mathcal{C})$ is hereditary if and only if whenever $0 \rightarrow C^{\prime} \rightarrow C \rightarrow C^{\prime \prime} \rightarrow 0$ is exact with $C, C^{\prime} \in \mathcal{C}$, then $C^{\prime \prime}$ is also in $\mathcal{C}$.

For a positive integer $n$, a left $R$-module $A$ is called an $n$-syzygy module (of a left $R$-module $M$ ) if there exists an exact sequence $0 \rightarrow A \rightarrow P_{n-1} \rightarrow \cdots \rightarrow P_{1} \rightarrow P_{0} \rightarrow M \rightarrow 0$ with all $P_{i}$ projective; a left $R$-module $B$ is said to be an $n$-cosyzygy module (of $M$ ) if there exists an exact sequence $0 \rightarrow M \rightarrow E^{0} \rightarrow E^{1} \rightarrow \cdots \rightarrow E^{n-1} \rightarrow B \rightarrow 0$ with all $E^{i}$ injective. 
Let $R$ be a ring. A submodule $T$ of a left $R$-module $N$ is called a pure submodule [4] if $0 \rightarrow A \otimes T \rightarrow A \otimes N$ is exact for all right $R$-modules $A$, or equivalently, if $\operatorname{Hom}(A, N) \rightarrow$ $\operatorname{Hom}(A, N / T) \rightarrow 0$ is exact for all finitely presented left $R$-modules $A$. An exact sequence $0 \rightarrow$ $T \rightarrow N \rightarrow N / T \rightarrow 0$ is called pure exact if $T$ is a pure submodule of $N$. A left $R$-module $M$ is said to be pure injective [2] if for every pure exact sequence $0 \rightarrow T \rightarrow N \rightarrow N / T \rightarrow 0$ of left $R$-modules, $0 \rightarrow \operatorname{Hom}(N / T, M) \rightarrow \operatorname{Hom}(N, M) \rightarrow \operatorname{Hom}(T, M) \rightarrow 0$ is exact.

Recall that a left $R$-module $M$ is called copure injective [2] if $\operatorname{Ext}_{R}^{1}(E, M)=0$ for all injective left $R$-modules $E$. A right $R$-module $M$ is said to be copure flat [3] if $\operatorname{Tor}_{1}^{R}(M, E)=0$ for all injective left $R$-modules $E$. These modules were introduced and studied by Enochs and Jenda $[2,3]$.

In [20], Yan, introduced the notion of Matlis injective modules as a generalization of copure injective modules and he proved that every module has Matis injective envelope over Noetherian rings. Recall that an $R$-module $M$ is said to be Matlis injective if $\operatorname{Ext}_{R}^{1}(E(R), M)=0$, where $E(R)$ denotes the injective envelope of $R$. Inspired by [3, 20], in this paper, we will introduce a concept of Matlis flat modules and investigate the existence of Matlis flat preenvelopes.

By [19, Lemma 5.1.1 and Proposition 5.1.2], every Gorenstein ring has flat injective hull. So, the rings with flat injective hulls may be generalization of Gorenstein rings. Also there exists a ring with flat injective hull which is not Gorenstein(see [6, Example 4.6]). Many authors characterized these rings (see for example [4, Theorem 9.3.3] and [8, Theorem 2.2]). Recently, Khosh-Ahang [9] extend these results and gather together 42 equivalent conditions for the commutative Noetherian rings with flat injective hull. In this paper, we give some more equivalent conditions for the injective envelope of commutative Artinian rings to be flat.

In Section 2, we give the definition of Matlis flat modules and present some of their general properties. An example is given to show that in general the class of all Matlis flat right $R$-modules properly contains the class of all copure flat right $R$-modules. We prove that if $R$ is a commutative Artinian ring and $S \subset R$ is a multiplicative set, then $S^{-1} M$ is a Matlis flat $S^{-1} R$-module for any Matlis flat $R$-module $M$. Also we give some equivalent conditions for the injective envelopes of rings to be flat.

In Section 3, we prove that if $R$ is a commutative Artinian ring, then every $R$-module has Matlis flat preenvelope and we give some equivalent conditions for every right $R$-module has a monic Matlis flat preenvelopes. Also we give some equivalent conditions for the injective envelope of commutative Artinian rings to be flat.

\section{Matlis flat modules}

We begin with the following definitions.

Definition 2.1. [20] Let $R$ be a ring and $M$ a left $R$-module. $M$ is said to be Matlis injective if $\operatorname{Ext}_{R}^{1}(E(R), M)=0$. A left $R$-module $N$ is said to be Matlis projective if $\operatorname{Ext} t_{R}^{1}(E(R), C)=0$ implies $\operatorname{Ext}_{R}^{1}(N, C)=0$ for any left $R$-module $C$. $R$ is said to be a left Matlis ring if $E(R)$ is flat and $p d_{R}(E(R)) \leq 1$.

Definition 2.2. Let $R$ be a ring. A right $R$-module $M$ is said to be Matlis flat if $\operatorname{Tor}_{1}^{R}(M, E(R))=$ 0 , where $E(R)$ denotes the injective envelope of $R$.

In what follows, we denote by $\mathcal{M F}$ the class of all Matlis flat right $R$-modules. 
Remark 2.3. We note that $\mathcal{M F}$ is closed under extensions and if $\left(M_{i}\right)_{i \in I}$ is a family of right $R$-modules, then $\bigoplus_{i \in I} M_{i}$ is Matlis flat if and only if each $M_{i}$ is Matlis flat.

Proposition 2.4. The following are hold for a ring $R$ :

1. $\mathcal{M F}=\operatorname{Mod}-R$ if and only if $E(R)$ is flat.

2. A right $R$-module $M$ is Matlis flat if and only if $M^{+}$is Matlis injective.

Proof. The assertion (1) follows by Definition 2.2 and the assertion (2) holds by the isomorphism: $\operatorname{Ext}_{R}^{1}\left(E(R), M^{+}\right) \cong \operatorname{Tor}_{1}^{R}(M, E(R))^{+}$.

Q.E.D.

We note that every copure flat right $R$-module is Matlis flat, but the converse is not true in general. The following example shows that there exist Matlis flat right $R$-modules which are not copure flat. Before that, we recall that a ring $R$ is said to be IF ring if every injective left $R$-module is flat.

Example 2.5. Consider a commutative non IF $\operatorname{ring} R$ for which $E(R)$ is flat, for example, let $R=\mathbb{Z}$ the ring of integers. Then, there exists an $R$-module which is not copure flat by [1, Corollary $4.3]$. Let $M$ be an $R$-module, but not copure flat. Since $E(R)$ is flat, $M$ is Matlis flat by Proposition 2.4 .

Proposition 2.6. The following are equivalent for a ring $R$ :

1. The flat dimension of $E(R)$ is at most 1 ;

2. Every submodule of a Matlis flat right $R$-module is Matlis flat;

3. Every submodule of a flat right $R$-module is Matlis flat;

4. Every submodule of a projective right $R$-module is Matlis flat.

Proof. $(1) \Rightarrow(2)$. Let $N$ be a submodule of a Matlis flat right $R$-module $M$. Then there exists an exact sequence $0 \rightarrow N \rightarrow M \rightarrow M / N \rightarrow 0$. Now applying the functor $\operatorname{Tor}_{1}^{R}(-, E(R))$ to this exact sequence, we have the exactness of

$$
\operatorname{Tor}_{2}^{R}(M / N, E(R)) \rightarrow \operatorname{Tor}_{1}^{R}(N, E(R)) \rightarrow \operatorname{Tor}_{1}^{R}(M, E(R)) .
$$

The first term is zero by (1), and the last term is zero since $M$ is Matlis flat. Consequently, $\operatorname{Tor}_{1}^{R}(N, E(R))=0$, and hence (2) holds.

$(2) \Rightarrow(3) \Rightarrow(4)$ is trivial.

$(4) \Rightarrow(1)$. For any right $R$-module $M$, there is an exact sequence $0 \rightarrow K \rightarrow P \rightarrow M \rightarrow 0$ with $P$ projective. Thus we get the exactness of $0 \rightarrow \operatorname{Tor}_{2}^{R}(M, E(R)) \rightarrow \operatorname{Tor}_{1}^{R}(K, E(R))$. The last term is zero by (4). Therefore $\operatorname{Tor}_{2}^{R}(M, E(R))=0$, which implies that the flat dimension of $E(R)$ is at most 1 .

Q.E.D.

Proposition 2.7. Let $R$ be a commutative Artinian ring and $S \subset R$ be a multiplicative set. If $M$ is a Matlis flat $R$-module, then $S^{-1} M$ is a Matlis flat $S^{-1} R$-module.

Proof. By [4, Theorem 2.1.11], we have the isomorphism

$$
S^{-1} \operatorname{Tor}_{1}^{R}\left(M, E_{R}(R)\right) \cong \operatorname{Tor}_{1}^{S^{-1} R}\left(S^{-1} M, S^{-1} E_{R}(R)\right) .
$$

But $S^{-1} E_{R}(R) \cong E_{S^{-1} R}\left(S^{-1} R\right)$ by [4, Theorem 3.3.3] and $R$ is a Noetherian ring. Thus $S^{-1} M$ is a Matlis flat $S^{-1} R$-module, when $\mathrm{M}$ is a Matlis flat $R$-module. 
Proposition 2.8. Every pure submodule of a Matlis flat right $R$-module is Matlis flat.

Proof. Let $M$ be a Matlis flat right $R$-module and $N$ a pure submodule of $M$. The pure exact sequence $0 \rightarrow N \rightarrow M \rightarrow M / N \rightarrow 0$ induces a split exact sequence $0 \rightarrow(M / N)^{+} \rightarrow M^{+} \rightarrow N^{+} \rightarrow$ 0. By Proposition 2.4, $M^{+}$is Matlis injective. Since $N^{+}$is isomorphic to a direct summand of $M^{+}$, $N^{+}$is Matlis injective by Remark 2.3. Therefore, $N$ is Matlis flat by Proposition 2.4 again. Q.E.D.

Proposition 2.9. The following are true for a commutative Artinian ring $R$ :

1. An $R$-module $M$ is Matlis injective if and only if $M^{+}$is Matlis flat.

2. An $R$-module $M$ is Matlis injective if and only if $M^{++}$is Matlis injective.

3. An $R$-module $M$ is Matlis flat if and only if $M^{++}$is Matlis flat.

4. $\mathcal{M F}$ is closed under direct products.

Proof. (1). Since $R$ is a commutative Artinian ring, $\operatorname{Tor}_{1}^{R}\left(M^{+}, E(R)\right)=0$ if and only if $\operatorname{Ext} t_{R}^{1}(E(R), M)^{+}=$ 0 for any $R$-module $M$ by the proof of [3, Lemma 3.6]. Hence the desired result follows.

(2). Let $M$ be a Matlis injective left $R$-module. Then $M^{+}$is Matlis flat by (1). Thus $M^{++}$is Matlis injective by Proposition 2.4. Conversely, if $M^{++}$is a Matlis injective left $R$-module, then $M$, as a pure submodule of $M^{++}$, is Matlis injective by [20, Proposition 3.13].

(3). If $M$ is a Matlis flat right $R$-module, then $M^{+}$is a Matlis injective left $R$-module, whence $M^{+++}$is Matlis injective by (2). Thus $M^{++}$is Matlis flat by Proposition 2.4. Conversely, if $M^{++}$is a Matlis flat right $R$-module, then $M$, being a pure submodule of $M^{++}$, is Matlis flat by Proposition 2.8.

(4). By hypothesis and [10, Theorem 3.64], $E(R)$ is finitely presented. Then $\mathcal{M F}$ is closed under direct products by [4, Theorem 3.2.26].

Q.E.D.

Proposition 2.10. Let $R$ be a ring. If $\operatorname{Tor}_{i}^{R}(M, E(R))=0$ for any $i$ with $1 \leq i \leq n+1$, then every $k$ th syzygy of $M$ is Matlis flat for $0 \leq k \leq n$.

Proof. Let $k$ be an integer with $0 \leq k \leq n$, and $C_{k}$ a $k$ th syzygy of $M$. Then there exists an exact sequence

$$
0 \rightarrow C_{k} \rightarrow P_{k-1} \rightarrow \cdots \rightarrow P_{1} \rightarrow P_{0} \rightarrow M \rightarrow 0,
$$

where each $P_{i}(0 \leq i \leq k-1)$ is projective. Then we have the isomorphism $\operatorname{Tor}_{1}^{R}\left(C_{k}, E(R)\right) \cong$ $\operatorname{Tor}_{k+1}^{R}(M, E(R))$. Note that $\operatorname{Tor}_{k+1}^{R}(M, E(R))=0$ by the hypothesis, it follows that $\operatorname{Tor}_{1}^{R}\left(C_{k}, E(R)\right)=$ 0 , and so $C_{k}$ is Matlis flat.

Q.E.D.

Proposition 2.11. Let $R$ be a ring. Then $\left(\mathcal{M F}, \mathcal{M F}^{\perp}\right)$ is a perfect cotorsion theory. Moreover the following are equivalent:

1. $\left(\mathcal{M F}, \mathcal{M F}^{\perp}\right)$ is a hereditary cotorsion theory;

2. $\operatorname{Tor}_{2}^{R}(F, E(R))=0$ for any $F \in \mathcal{M F}$;

3. $\operatorname{Tor}_{j}^{R}(F, E(R))=0$ for any $F \in \mathcal{M F}$ and any $j \geq 1$.

Proof. By [18, Lemma 1.11 and Theorem 2.8] $\left(\mathcal{M F}, \mathcal{M F}^{\perp}\right)$ is a perfect cotorsion theory.

$(1) \Rightarrow(2)$. For any Matlis flat module $F$, there is an exact sequence $0 \rightarrow K \rightarrow P \rightarrow F \rightarrow 0$

with $P$ projective. Then $K$ is Matlis flat by (1), and so $\operatorname{Tor}_{2}^{R}(F, E(R)) \cong \operatorname{Tor}_{1}^{R}(K, E(R))=0$.

$(2) \Rightarrow(3)$. Let $F$ be a Matlis flat module. Then $\operatorname{Tor}_{1}^{R}(F, E(R))=0$ by definition and $\operatorname{Tor}_{j}^{R}(F, E(R))=0$ for any $j \geq 2$ by induction. 
$(3) \Rightarrow(1)$ is easy.

Q.E.D.

Recall that a ring $R$ is called right semi-artinian[17] if every non-zero right $R$ module has non-zero socle.

Theorem 2.12. The following are equivalent for a ring $R$ :

1. Every right $R$-module is Matlis flat;

2. $E(R)$ is flat;

3. Every cotorsion left $R$-module is Matlis injective;

4. Every pure injective left $R$-module is Matlis injective;

5 . Every finitely presented right $R$-module is Matlis flat;

6. Every right $R$-module $M$ with $M \in \mathcal{M F}^{\perp}$ is injective.

If $R$ is right semi-artinian, then the above conditions are equivalent to:

7. Every simple right $R$-module is Matlis flat.

Proof. (1) $\Leftrightarrow(2)$ follows from Proposition 2.4.

$(2) \Rightarrow(3)$. Let $M$ be a cotorsion left $R$-module. Then $\operatorname{Ext}_{R}^{1}(E(R), M)=0$, since $E(R)$ is flat by (2). Hence $M$ is Matlis injective.

$(3) \Rightarrow(4)$. It follows from the fact that every pure injective left $R$-module is cotorsion.

$(4) \Rightarrow(1)$. Let $M$ be a right $R$ module. Then $M^{+}$is pure injective, and so $M^{+}$is Matlis injective by (4). It follows that $M$ is Matlis flat by Proposition 2.4.

$(1) \Rightarrow(5),(7)$ are trivial.

$(5) \Rightarrow(2)$. We have $\operatorname{Tor}_{1}^{R}(M, E(R))=0$ for any finitely presented right $R$-module $M$ by (5). Therefore $E(R)$ is flat, as desired.

(1) $\Leftrightarrow(6)$ follows from Proposition 2.11.

$(7) \Rightarrow(2)$. Let $I$ be a maximal right ideal of $R$. Then we have $\operatorname{Tor}_{1}^{R}(R / I, E(R))=0$ by (7). Thus $\operatorname{Ext}_{R}^{1}\left(R / I, E(R)^{+}\right)=0$ since $\operatorname{Ext}_{R}^{1}\left(R / I, E(R)^{+}\right)=\operatorname{Tor}_{1}^{R}(R / I, E(R))^{+}$. So $E(R)^{+}$is injective with respect to any maximal right ideal of $R$. Hence $E(R)^{+}$is injetive by [16, Lemma 4] since $R$ is right semi-artinian. Thus $E(R)$ is flat.

Q.E.D.

By Definition 2.1 and Theorem 2.12, we have the following corollary.

Corollary 2.13. Let $R$ be a ring with $p d(E(R)) \leq 1$. Then the following are equivalent:

1. $R$ is a left Matlis ring;

2. Every right $R$-module is Matlis flat;

3. Every cotorsion left $R$-module is Matlis injective;

4. Every pure injective left $R$-module is Matlis injective;

5. Every finitely presented right $R$-module is Matlis flat;

6. Every right $R$-module $M$ with $M \in \mathcal{M F}^{\perp}$ is injective.

Proposition 2.14. Let $R$ be a commutative ring and $S$ a simple $R$-module. Then the following are equivalent:

1. $S$ is Matlis injective;

2. $S$ is Matlis flat;

3. $S^{+}$is Matlis injective.

Proof. $(1) \Leftrightarrow(2)$. It follows from the proof of [13, Proposition 2.8].

$(2) \Leftrightarrow(3)$ follows from Proposition 2.4. 


\section{Matlis flat (pre)envelopes and Matlis injective covers}

For $\mathcal{C}=\mathcal{M F}, \mathcal{C}$-(pre)envelopes and $\mathcal{C}$-(pre)covers will simply be called Matlis flat (pre)envelopes and Matlis flat (pre)covers.

Theorem 3.1. If $R$ is a commutative Artinian ring, then every $R$-module has Matlis flat preenvelope.

Proof. Let $M$ be an $R$-module. By [4, Lemma 5.3.12], there is an infinite cardinal number $\aleph_{\alpha}$ such that for any homomorphism $f: M \rightarrow N$ with $N$ Matlis flat, there is a pure submodule $P$ of $N$ such that $\operatorname{Card}(P) \leq \aleph_{\alpha}$ and $f(M) \subseteq P$. Note that $P$ is Matlis flat by Proposition 2.8. By Proposition 2.9 , the product of Matlis flat $R$-modules is Matlis flat and hence $M$ has a Matlis flat preenvelope by Enochs and Jenda [4, Proposition 6.2.1].

Q.E.D.

Proposition 3.2. Let $R$ be a commutative Artinian ring.

1. If $f: C \rightarrow D$ is a Matlis flat preenvelope of a module $C$ in Mod- $R$, then $f^{+}: D^{+} \rightarrow C^{+}$is a Matlis injective precover of $C^{+}$in $R$-Mod.

2. If $f: C \rightarrow D$ is a Matlis injective preenvelope of a module $C$ in $R$-Mod, then $f^{+}: D^{+} \rightarrow C^{+}$ is a Matlis flat precover of $C^{+}$in $R$-Mod.

Proof. By Proposition 2.4 and Proposition 2.9, we have $\mathcal{M F}^{+} \subseteq \mathcal{M I}$ and $\mathcal{M I}^{+} \subseteq \mathcal{M F}$. Now both the assertions follows immediately from [6, Theorem 3.1].

In what follows, we discuss when every right $R$-module has a monic Matlis flat preenvelope.

Theorem 3.3. The following are equivalent for a commutative Artinian ring $R$ :

1. $R$ is Matlis injective as an $R$-module;

2. Every $R$-module has a monic Matlis flat preenvelope;

3. Every injective $R$-module is Matlis flat;

4. Every flat $R$-module is Matlis injective;

5 . Every $R$-module has an epic Matlis injective cover;

6 . Every $R$-module is a submodule of a Matlis flat $R$-module

Proof. (1) $\Rightarrow(2)$. Let $M$ be an $R$-module. By Theorem 3.1, $M$ has a Matlis flat preenvelope $f: M \rightarrow F$. Since $R^{+}$is a cogenerator in the category of $R$-modules, there is an exact sequence $0 \rightarrow M \rightarrow \prod R^{+}$. Note that $R^{+}$is a Matlis flat $R$-module by assumption and Proposition 2.9. It follows that $\prod R^{+}$is Matlis flat by Proposition 2.9. Thus $f$ is monic, and so (2) follows.

$(2) \Rightarrow(3)$. Let $E$ be an injective $R$-module. By (2), there exists an exact sequence $0 \rightarrow E \rightarrow$ $F \rightarrow N \rightarrow 0$, where $E \rightarrow F$ is a Matlis flat preenvelope with $F$ Matlis flat. Then this short exact sequence is split since $E$ is injective. Thus $E$ is Matlis flat as a direct summand of $F$ by Remark 2.3. Hence (3) holds.

(3) $\Rightarrow(4)$. Let $M$ be a flat $R$-module. Then $M^{+}$is injective, and so $M^{+}$is Matlis flat by assumption. So $M$ is Matlis injective by Proposition 2.9, as desired.

$(4) \Rightarrow(1)$ is trivial.

$(1) \Rightarrow(5)$. Let $M$ be an $R$-module. Then $M$ has a Matlis injective cover $g: E \rightarrow M$ by $[20$, Remark 4.5]. On the other hand, there exists an exact sequence $F \rightarrow M \rightarrow 0$ with $F$ free. Note that $F$ is Matlis injective by (1) and [20, Proposition 3.13], so $g$ is an epimorphism. 
$(5) \Rightarrow(1)$. Because there exists an epic Matlis injective cover $f: E \rightarrow R$ by (5), $R$ is isomorphic to a direct summand of $E$. Thus $R$ is Matlis injevtive by [20, Proposition 3.13].

$(4) \Rightarrow(6)$ is trivial.

$(6) \Rightarrow(4)$. By Theorem 3.1, every $R$-module has a Matlis flat preenvelope. Thus the assertion holds by $(6)$.

Q.E.D.

Recall that a $\mathcal{C}$-envelope $\varphi: M \rightarrow C$ is said to have unique mapping property if for any homomorphism $f: M \rightarrow C^{\prime}$ with $C^{\prime} \in \mathcal{C}$, there is a unique homomorphism $g: C \rightarrow C^{\prime}$ such that $g \varphi=f$. Dually we have the definition of $\mathcal{C}$-cover with unique mapping property.

Theorem 3.4. The following are equivalent for a commutative Artinian ring $R$ :

1. $E(R)$ is flat;

2. The projective dimension of $E(R)$ is at most 1 ;

3. The projective dimension of $E(R)$ is at most 2 ;

4. Every $R$-module has an epic Matlis flat preenvelope;

5. Every cotorsion $R$-module has an epic Matlis flat preenvelope;

6. Every $R$-module has a monic Matlis injective cover;

7. Every $R$-module has a Matlis injective cover with the unique mapping property;

8. Every $R$-module has a Matlis flat envelope with the unique mapping property;

9. The cokernel of any Matlis injective preenvelope of an $R$-module is Matlis injective;

10. The cokernel of any Matlis flat precover of an $R$-module is Matlis flat.

Proof. $(1) \Leftrightarrow(2) \Leftrightarrow(3)$ follows from [8, Theorem 2.2] and the fact that $E(R)$ is finitely generated.

$(2) \Rightarrow(4)$. By Theorem 3.1, every $R$-module $M$ has a Matlis flat preenvelope $f: M \rightarrow N$. Note that $i m(f)$ is Matlis flat by Proposition 2.6, hence $f: M \rightarrow i m(f)$ is an epic Matlis flat preenvelope.

$(4) \Rightarrow(5)$ is trivial.

$(5) \Rightarrow(2)$. By Proposition 2.6, it is enough to prove that any submodule $N$ of any Matlis flat $R$-module $M$ is Matlis flat. Since $M / N$ has a flat cover $f: F \rightarrow M / N$, we get an exact sequence $0 \rightarrow C \rightarrow F \rightarrow M / N \rightarrow 0$ with $C$ cotorsion by Wakamatsu's Lemma. By (5), $C$ has an epic Matlis flat preenvelope. Thus $C$ is Matlis flat since $C$ embeds in a flat $R$-module. So we get an induced exact sequence

$$
0=\operatorname{Tor}_{2}^{R}(F, E(R)) \rightarrow \operatorname{Tor}_{2}^{R}(M / N, E(R)) \rightarrow \operatorname{Tor}_{1}^{R}(C, E(R))=0 .
$$

Hence $\operatorname{Tor}_{2}^{R}(M / N, E(R))=0$. On the other hand, the short exact sequence $0 \rightarrow N \rightarrow M \rightarrow$ $M / N \rightarrow 0$ induces the exactness of the sequence

$$
0=\operatorname{Tor}_{2}^{R}(M / N, E(R)) \rightarrow \operatorname{Tor}_{1}^{R}(N, E(R)) \rightarrow \operatorname{Tor}_{1}^{R}(M, E(R))=0
$$

Therefore $\operatorname{Tor}_{1}^{R}(N, E(R))=0$, as desired.

$(2) \Leftrightarrow(6)$. By [20, Proposition 3.13], the class of all Matlis injective $R$-modules is closed under direct sums. So $(2) \Leftrightarrow(6)$ follows from [20, Proposition 3.7] and [11, Proposition 4].

$(3) \Rightarrow(7)$. Let $M$ be any $R$-module. Then $M$ has a Matlis injective cover by [20, Remark 4.5]. It is enough to show that, for any Matlis injective $R$-module $G$ and any homomorphism $g: G \rightarrow F$ such that $f g=0$, we have $g=0$. In fact, there exists $\beta: F / i m(g) \rightarrow M$ such that $\beta \pi=f$ since $i m(g) \subseteq \operatorname{ker}(f)$, where $\pi: F \rightarrow F / i m(g)$ is the natural map. Since projective dimension of $E(R)$ is 
at most 2, $\operatorname{Ext}_{R}^{i}(E(R), \operatorname{ker}(g))=0$ for any $i \geq 3$. It follows that $F / i m(g)$ is Matlis injective. Thus there exists $\alpha: F / i m(g) \rightarrow F$ such that $\beta=f \alpha$, and so we get the exact commutative diagram.

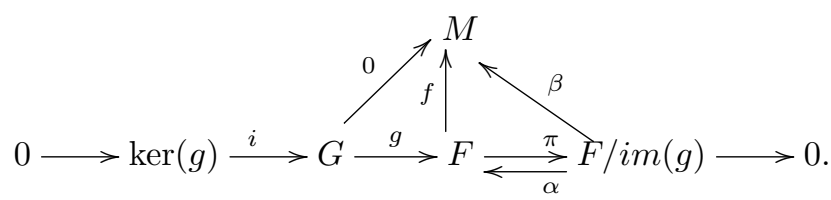

Thus $f \alpha \pi=f$, and hence $\alpha \pi$ is an isomorphism. Therefore $\pi$ is monic and so $g=0$.

$(7) \Rightarrow(3)$. Let $M$ be any $R$-module. Then we have the exact sequence

$$
0 \rightarrow M \rightarrow E^{0} \stackrel{\varphi}{\rightarrow} E^{1} \stackrel{\psi}{\rightarrow} N \rightarrow 0
$$

where $E^{0}, E^{1}$ are injective. Let $\theta: H \rightarrow N$ be a Matlis injective cover with unique mapping property. Then there exists $\tau: E^{1} \rightarrow H$ such that $\psi=\theta \tau$. Thus $\theta \tau \varphi=\psi \varphi=0=\theta 0$, and hence $\tau \varphi=0$, which implies that $\operatorname{ker}(\psi)=i m(\varphi) \subseteq \operatorname{ker}(\tau)$. Therefore there exists $\gamma: N \rightarrow H$ such that $\gamma \psi=\tau$, and so we get the exact commutative diagram:

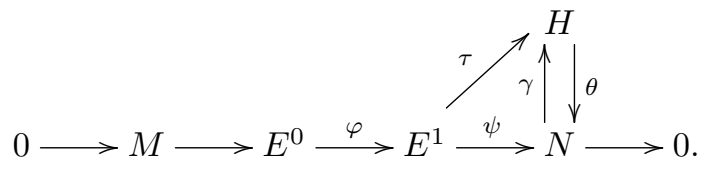

Thus $\theta \gamma \psi=\psi$, and so $\theta \gamma=1_{N}$ since $\psi$ is epic. It follows that $N$ is isomorphic to a direct summand of $H$, and hence $N$ is Matlis injective. Now applying the functor $\operatorname{Hom}_{R}(E(R),-)$ to the above exact sequence, we get $\operatorname{Ext}_{R}^{3}(E(R), M) \cong \operatorname{Ext}_{R}^{1}(E(R), N)=0$. Consequently $E x t_{R}^{3}(E(R), M)=0$ as desired.

$(3) \Leftrightarrow(8)$. It is similar to the proof of $(3) \Leftrightarrow(7)$.

$(2) \Leftrightarrow(9)$ is trivial.

$(4) \Rightarrow(10)$. Let $g: M \rightarrow F$ be a Matlis flat preenvelope of an $R$-module $M$ with $\operatorname{Coker}(g)=L$. Since there exists an epic Matlis flat preenvelope $g^{\prime}: M \rightarrow F^{\prime}$ by (4), we have $L \oplus F^{\prime} \cong F$ by the dual of [4, Lemma 8.6.3]. Hence by Remark 2.3, $L$ is Matlis flat as desired.

$(10) \Rightarrow(2)$. By Proposition 2.6, it is enough to prove that any submodule of a Matlis flat $R$-module is Matlis flat. Let $M$ be a submodule of a Matlis flat $R$-module $M$. Note that $N$ has a Matlis flat preenvelope $f: N \rightarrow F$, it follows that $f$ is a monomorphism. By (10), $\operatorname{Coker}(f)$ is Matlis flat. So we have the exact sequence $0 \rightarrow M \rightarrow F \rightarrow \operatorname{Coker}(f) \rightarrow 0$. Applying the functor $\operatorname{Tor}_{1}^{R}(-, E(R))$ to this exact sequence we get $\operatorname{Tor}_{1}^{R}(N, E(R))=0$ and hence $N$ is Matlis flat.

Q.E.D.

Theorem 3.5. Let $R$ be a commutative Artinian ring and $S \subset R$ be a multiplicative set. Then:

1. If $\varphi: C \rightarrow M$ is a Matlis injective cover of $M$ as an $S^{-1} R$-module, then $\varphi: C \rightarrow M$ is also a Matlis injective cover of $M$ as an $R$-module. 
2. If $\psi: D \rightarrow M$ is a Matlis flat cover of $M$ as an $S^{-1} R$-module, then $\psi: D \rightarrow M$ is also a Matlis flat cover of $M$ as an $R$-module.

Proof. (1). Let $N$ be any Matlis injective $R$-module. Then $S^{-1} N$ is a Maltis injective $S^{-1} R$-module by [20, Proposition 3.14]. For any $R$-homomorphism $f: N \rightarrow M$, there is an $S^{-1} R$-homomorphism $g: S^{-1} N \rightarrow M$ such that $g \alpha=f$ by [14, Proposition 3.9], where $\alpha: N \rightarrow S^{-1} N$ is the canonical map. Thus there exists $\beta: S^{-1} N \rightarrow C$ such that $g=\varphi \beta$ since $\varphi$ is an Matlis injective precover of $M$ as an $S^{-1} R$-module. Therefore, $\varphi(\beta \alpha)=(\varphi \beta) \alpha=g \alpha=f$, and so $\varphi$ is a Matlis injective precover of $M$ as an $R$-module. Let $h: C \rightarrow C$ be an $R$-homomorphism with $\varphi h=\varphi$. Note that $h$ is also an $S^{-1} R$-homomorphism by [15, Exercise 3.50] since $C$ is an $S^{-1} R$-module. Thus $h$ is an automorphism, and so $\varphi$ is an Matlis injective cover of $M$ as an $R$-module.

(2). The proof is similar to that of (1).

Q.E.D.

Theorem 3.6. The following are equivalent for a ring $R$ :

1. The injective envelope $E(M)$ is Matlis flat for any Matlis flat right $R$-module $M$;

2. The Matlis flat cover $F(I)$ is injective for any injective right $R$-module $I$.

Proof. $(1) \Rightarrow(2)$. Let $I$ be an injective right $R$-module, $f: F(I) \rightarrow I$ the Matlis flat cover of $I$, and $g: F(I) \rightarrow E(F(I))$ the injective envelope. Then there exists $h: E(F(I)) \rightarrow I$ such that $h g=f$. On the other hand, since $E(F(I))$ is Matlis flat by (1), there exists $j: E(F(I)) \rightarrow F(I)$ such that $f j=h$. Thus $f j g=f$, and hence $j g$ is an isomorphism. This means that $F(I)$ is a direct summand of $E(F(I))$ and so it is injective.

$(2) \Rightarrow(1)$. Let $M$ be a Matlis flat right $R$-module, $\alpha: M \rightarrow E(M)$ the injective envelope, and $\beta: F(E(M)) \rightarrow E(M)$ the Matlis flat cover of $E(M)$. Then there exists $\gamma: M \rightarrow F(E(M))$ such that $\beta \gamma=\alpha$. On the other hand, since $F(E(M))$ is injective by (2), there exists $\delta: E(M) \rightarrow$ $F(E(M))$ such that $\delta \alpha=\gamma$. Thus $\beta \delta \alpha=\alpha$ and so $\beta \delta$ is an isomorphism. It follows that $E(M)$ is Matlis flat.

Q.E.D.

\section{Acknowledgments}

The authors thank Professor Hangyu Yan for reading the manuscript and providing his helpful remarks.

\section{References}

[1] N. Ding and J. Chen, On copure flat modules and flat resolvents, Comm. Algebra 24(3) (1996), 1071-1081.

[2] E. E. Enochs and O. M. G. Jenda, Copure injective modules, Quaest. Mathematicae 14 (1991), 401-409.

[3] E. E. Enochs and O. M. G. Jenda, Copure injective resolutions, flat resolvents and dimensions, Comment. Math. Univ. Carolin. 34, 2 (1993), 203-211.

[4] E. E. Enochs and O. M. G. Jenda, Relative Homological Algebra, De Gruyter Expositions in Mathematics No. 30, New York, 2000. 
[5] E. E. Enochs, O.M.G. Jenda, J.A. Lopez-Ramos, The existence of Gorenstein flat covers, Math. Scand. 94 (2004), 46-62.

[6] E. E. Enochs, Z. Y. Huang, Injective envelopes and (Gorenstein) flat covers, Algebr. Represent. Theory 15 (2012), 11311145.

[7] L. Fuchs, L. Salce, Modules over Non-Noetherian Domains, Mathematical Surveys and Monographs, 84, American Mathematical Society, Providence, RI, 2001.

[8] K. Khashyarmanesh and Sh. Salarian, On the rings whose injective hulls are flat, Proc. Amer. Math. Soc. 131 (2003), 2329-2335.

[9] F. Khosh-Ahang, A homological view on rings with flat injective hulls, Arch. Math. 105 (2015), 23-32.

[10] T. Y. Lam, Lectures on Modules and Rings, Grad. Texts in Math. 189, Springer, New York, 1999.

[11] J. R. Garcia Rozas, B. Torrecillas, Relative injective covers, Comm. Algebra, 22 (1994), 29252940.

[12] R. Gobel and J. Trlifaj, Approximations and Endomorphisms Algebras of Modules, de Gruyter, Berlin, 2000.

[13] L. X. Mao and N. Q. Ding, Relative copure injective and copure flat modules, J. Pure Appl. Algebra, 208(2) (2007), 635-646.

[14] K. Pinzon, Absolutely pure modules, Ph.D thesis, University of Kentucky, 2005.

[15] J. J. Rotman, An Introduction to Homological Algebra, Academic press, New York, 1979.

[16] P. F. Smith, Injective modules and prime ideals, Comm. Algebra, 9 (1981), 989-999.

[17] B. Stenström, Rings of Quotients, Springer-Verlag, Berlin, Heidelberg, New York, 1975.

[18] J. Trlifaj, Covers, Envelopes, and Cotorsion Theories; Lecture notes for the workshop, "Homological Methods in Module Theory", Cortona, September 10-16, 2000.

[19] J. Xu, Flat covers of modules, Lecture Notes in Mathematics, 1634, Springer-Verlag, Berlin, 1996.

[20] H. Yan, Matlis injective modules, Bull. Korean Math. Soc., 50 (2013), 459-467. 